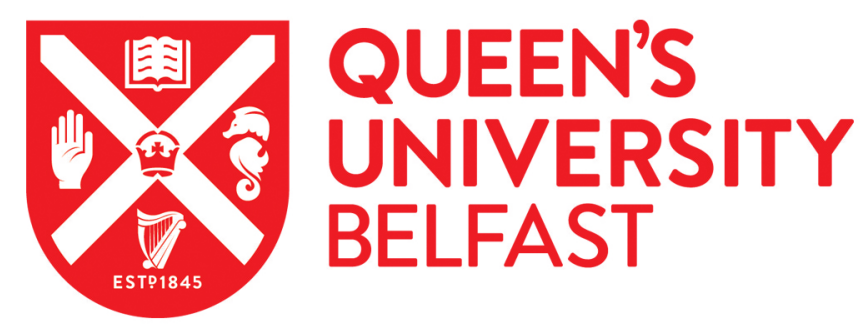

\title{
123I-FP-CIT striatal binding ratios do not decrease significantly with age in older adults
}

Roberts, G., Lloyd, J. J., Petrides, G. S., Donaghy, P., Kane, J., Durcan, R., Lawley, S., Howe, K., Sims, A., Taylor, J-P., O'Brien, J. T., \& Thomas, A. J. (2019). 123I-FP-CIT striatal binding ratios do not decrease significantly with age in older adults. Annals of Nuclear Medicine. https://doi.org/10.1007/s12149-019-01352-x

Published in:

Annals of Nuclear Medicine

Document Version:

Peer reviewed version

Queen's University Belfast - Research Portal:

Link to publication record in Queen's University Belfast Research Portal

Publisher rights

(c) The Japanese Society of Nuclear Medicine 2019. This work is made available online in accordance with the publisher's policies. Please refer to any applicable terms of use of the publisher.

\section{General rights}

Copyright for the publications made accessible via the Queen's University Belfast Research Portal is retained by the author(s) and / or other copyright owners and it is a condition of accessing these publications that users recognise and abide by the legal requirements associated with these rights.

Take down policy

The Research Portal is Queen's institutional repository that provides access to Queen's research output. Every effort has been made to ensure that content in the Research Portal does not infringe any person's rights, or applicable UK laws. If you discover content in the Research Portal that you believe breaches copyright or violates any law, please contact openaccess@qub.ac.uk. 


\section{${ }^{123}$ I-FP-CIT striatal binding ratios do not decrease significantly with age in older adults}

Gemma Roberts $^{1,2}$, James J. Lloyd ${ }^{1,2}$, George S. Petrides ${ }^{2}$, Paul C. Donaghy ${ }^{1}$, Joseph PM Kane ${ }^{1,3}$, Rory Durcan ${ }^{1}$, Sarah Lawley ${ }^{1}$, Kim Howe ${ }^{2}$, Andrew J Sims ${ }^{4,5}$ John-Paul Taylor $^{1}$, John T. O’Brien ${ }^{1,6}$, Alan J. Thomas ${ }^{1}$

1: Institute of Neuroscience, Newcastle University, Biomedical Research Building, Campus for Ageing and Vitality, Newcastle upon Tyne, NE4 5PL, United Kingdom

2: Nuclear Medicine department, Royal Victoria Infirmary, Newcastle upon Tyne NHS Foundation Hospitals Trust, Newcastle upon Tyne, United Kingdom, NE1 4LP

3: Centre for Public Health, Queen's University Belfast, Institute of Clinical Sciences, Block B, Royal Victoria Hospital, Belfast, BT12 6BA, United Kingdom

4: Northern Medical Physics and Clinical Engineering, Freeman Hospital, Newcastle upon Tyne NHS Foundation Hospitals Trust, Newcastle upon Tyne, NE7 7DN, United Kingdom

5: Institute of Cellular Medicine, Faculty of Medical Science, Newcastle University, Newcastle upon Tyne, NE2 4HH, United Kingdom

6: Department of Psychiatry, University of Cambridge, Cambridge Biomedical Campus, Cambridge, CB2 OSP, United Kingdom

CORRESPONDING AUTHOR:

Gemma Roberts

Institute of Neuroscience

Biomedical Research Building

Campus for Ageing and Vitality

Newcastle University

Newcastle upon Tyne

NE4 5PL

Email gemma.roberts@newcastle.ac.uk

Telephone: +447890489690 (personal number, email preferred)

Article type: Original article

Short title: FP-CIT age-dependency in old age

Funding sources for study:

The lead author is receiving full-time support from the Alzheimer's Society via a Clinical Training Fellowship. The clinical studies used in this project were funded by Alzheimer's Research UK and the Newcastle National Institute for Health Research (NIHR) Biomedical Research Centre, hosted by Newcastle upon Tyne Hospitals NHS Foundation Trust and Newcastle University. 


\section{Abstract}

Objective

I-123-2 $\beta$-Carbomethoxy-3 $\beta$-(4-iodophenyl)- $\mathrm{N}$-(3-fluoropropyl)nortropane (FP-CIT) imaging is an established biomarker used in the diagnosis of Lewy body disease. Images are often reported with the aid of striatal binding ratios (SBRs), comparing uptake to a normal database via $Z$ scores.

It is well-known that SBRs are age-dependent. However, previous studies cover wide age ranges between 20 and 80 years, rather than focusing on older adults. Typically a linear relationship is reported, but some authors have suggested that SBRs do not decline as rapidly in old age.

Commercial software packages usually adjust the SBR Z score to attempt to compensate for agerelated decline, but the model used varies. Ensuring age-correction is appropriate for older adults is important, given that the majority of patients referred for FP-CIT scans are aged over 60 years. We examined the relationship of SBR with age in older adults and the effect of age correction using research scans from 123 adults over 60 years of age.

Methods

Twenty-nine healthy older adults and twenty-three with $\mathrm{MCl}$ due to Alzheimer's disease were included as controls, i.e. individuals with no evidence of Lewy body disease. Their ages ranged from 60 - 92 years (mean 76; SD 7.9)). SBRs and Z scores were calculated using BRASS (Hermes Medical) and DaTQUANT (GE Healthcare). SBRs were plotted against age and linear mixed effects models applied. We tested the effect of removing age-correction in BRASS using an independent dataset of 71 older adults with dementia or mild cognitive impairment.

Results

The slopes of the linear fits between SBR and age per year were $-0.007(p=0.30)$ with BRASS and $-0.004(p=0.35)$ with DaTQUANT. The slopes are smaller than reported in the literature and show no statistically significant difference from zero.

Switching age correction off in BRASS in the test subjects reduced Z scores by approximately 1 standard deviation at 80 years of age.

Conclusion

We found no statistically significant age-related decline in SBR in adults over 60 years of age without Lewy body disease. Commercial software packages that apply a fixed rate of age correction may be overcorrecting for age in older adults, which could contribute to misdiagnosis.

\section{Keywords}

FP-CIT, DaTSCAN, SBR, age-dependency, Lewy body disease 


\section{Introduction}

${ }^{123}$-2 $\beta$-Carbomethoxy-3 $\beta$-(4-iodophenyl)- $\mathrm{N}$-(3-fluoropropyl) nortropane (FP-CIT) SPECT imaging (DaTSCAN ${ }^{\mathrm{TM}}$ ) is a well-established dopamine transporter molecular imaging technique for pre-synaptic dopaminergic function. It is approved by the FDA and EU for the differential diagnosis of Parkinson's disease (PD) from essential tremor and dementia with Lewy bodies (DLB) from other causes of dementia and is included as an indicative biomarker in the latest diagnostic guidelines for DLB [1].

The introduction of sophisticated fully automated commercial ${ }^{123}$ I-FP-CIT software packages [2-6], combined with greater awareness in the nuclear medicine community of "balanced loss" of dopaminergic function, particularly in DLB, has increased interest in quantification for FP-CIT imaging [7]. Balanced loss can be challenging for the non-expert to identify visually as images are scaled to maximum uptake rather than to a predefined absolute uptake level; low striatal binding ratio (SBR) values for striata with otherwise normal appearance are therefore useful for identifying global loss of uptake. Z scores, defined as the number of standard deviations the subject SBR is away from the mean SBR of a database of healthy control subjects, are often used to help show how normal or abnormal an SBR value is, with a Z score below -2 typically raising concern for an abnormal scan.

Studies in healthy controls over a wide range of ages have demonstrated that striatal uptake levels decrease with age, confirmed by Karrer et al. in their 2017 meta-analysis [8]. It is therefore important to interpret striatal binding ratios by comparing with age-matched healthy controls and $Z$ scores are commonly age-adjusted for this purpose. However, the exact form of the relationship between SBR and age is not clear, leading commercial software packages to implement different age correction models. Patients referred for FP-CIT scans for dementia diagnosis are typically over 60 years of age and often much older. It is therefore vital to ensure that age-correction is appropriate for this age group.

Some dopamine transporter availability studies report a linear decline in SBR with age, for example the ENC-DAT study [9]. This is a large European initiative led by the European Association of Nuclear Medicine (EANM), which included FP-CIT scans of 139 adults aged between 20 and 83 years and found slopes (absolute change in SBR per year) of -0.015 (95\% confidence interval: -0.021 to -0.009$)$ for men and -0.018 ( -0.025 to -0.011$)$, which they state corresponds to a 4 to $7 \%$ decline in SBR per decade depending on the striatal region assessed [9]. Prior to this, a large single-center study of 186 adults found a loss of $46 \%$ over $18-88$ years, on average $6.6 \%$ per decade, also applying a linear model [10]. A recent publication studying 256 healthy individuals in Japan aged between 30 and 83 [11] demonstrated a linear fit for both males and females, reporting an average decline of $6.3 \%$ per decade. However, a study using a Tc-99m ligand similar to FP-CIT [12] concluded that an exponential or bi-linear function of SBR with age is a better model, with the decline tailing off in older age. The meta-analysis by Karrer et al. [8], which combined data on a variety of PET and SPECT dopamine transporter ligands, concluded that while there is some suggestive evidence for steeper decline in young adults, a linear relationship describes the decrease in function with age as well as an exponential function (coefficient of determination $\left(R^{2}\right)$ values of $38.6 \%$ for linear fit and $37.1 \%$ for log-linear fit). However, most subjects were not scanned with ${ }^{123} \mathrm{I}-\mathrm{FP}-\mathrm{ClT}$, with only 5 such studies included 
within the 33 studies on dopamine transporter availability. In addition, the majority of the 289 ${ }^{123}$ I-FP-CIT scans included appear to come from two studies published by the ENC-DAT team [4, 13] suggesting that double counting may have occurred. The relationship in older subjects alone was not studied, as this was not the focus of the meta-analysis, so it was not demonstrated that a significant age-dependency exists in this age group.

Two commercial software packages were used in the current study: DaTQUANT v1.01 standalone software (GE Healthcare, Little Chalfont, UK) and Hybrid Viewer BRASS v2.5 (Hermes Medical Ltd, Stockholm, Sweden). The DaTQUANT normal database consists of 119 healthy control subjects (aged 30 - 84 years) taken from the Parkinson's Progression Marker Initiative study (PPMI https://www.ppmi-info.org [14]). A bi-linear model with different slopes for individuals under 55 and over 68 , with a transition region in between is implemented. This was generated by the DaTQUANT developers by splitting the control SBRs into two clusters with different linear fits, since it was noticed that the SBR does not change as rapidly in older age (Personal communication, GE Healthcare). This was done separately for the left and right striata and for each sub-region resulting in small differences between regions and sides. The slopes implemented for the whole striatum (i.e. absolute change in SBR per year) are -0.025 for both striata under 55 years of age and -0.012 and -0.007 for the left and right striata respectively over 68 years (Personal communication, GE Healthcare). The DaTQUANT SBR intercepts (i.e. projected SBR at age zero) are 3.5 (left striatum) and 3.4 (right) for the younger cluster and 2.7 (left) and 2.3 (right) for the older cluster. These slopes and SBR intercept values apply to images reconstructed with OSEM iterative reconstruction without attenuation correction, and correspond to decreases in SBR of $0.25(10 \%)$ between 40 and 50 years, $0.12(7 \%)$ between 70 and 80 years for the left striatum and 0.07 (4\%) between 70 and 80 years for the right striatum. A bi-linear model can also be implemented within BRASS, but we used the default model, which is a linear fit to 103 of the controls from the ENC-DAT study aged between 21 and 83 years, used as the healthy control database in BRASS.

Our experience with both software packages has been that SBRs for the oldest adults can be quite low and the scans can have abnormal visual appearances, yet the age-corrected $Z$ scores can be within the normal range. This raises the possibility that the age correction required for older people may be overestimated. Published data showing SBR against age for the healthy controls from the ENC-DAT study [9], which applied a single linear fit to all ages, shows little evidence of a clear continuing decrease in SBR with age in adults over 60 years old, substantial variation between subjects and relatively few data points for subjects over 75 years. The DaTQUANT database also shows no convincing decline in SBR with age for subjects over 60 (SBR data accessed within the software).

We recruited healthy older adults and older adults with cognitive impairment but no signs of Lewy body disease to determine whether there is evidence of age-dependency of SBR in adults over 60 years of age. We also used a separate independent test group of individuals over 60 with and without Lewy body disease to test our findings concerning age correction. 


\section{Methods}

\section{Subject selection}

Healthy older adults, adults with mild cognitive impairment $(\mathrm{MCl})$ and adults with dementia were taken from three Newcastle University studies. All were over 60 years of age and provided written informed consent, including for their data to be used in more than one research study. The healthy older adults and the $\mathrm{MCI}-\mathrm{AD}$ individuals from one of the studies were combined to give a large group of controls (i.e. older people without evidence of Lewy body disease). An independent group of individuals with $\mathrm{MCl}$ and individuals with dementia was used to test the effects of age correction. For both the control and test groups, MRI brain scans and CT scans (where available) were reviewed by a radiologist to exclude other causes of cognitive impairment.

\section{Control group: $\mathrm{MCl}-\mathrm{AD}$ and healthy older adults}

Twenty-six individuals meeting the NIA-AA clinical criteria for $\mathrm{MCl}$ due to Alzheimer's disease (MCI-AD) [15] were recruited from memory clinics, specialist dementia services, elderly medicine clinics and neurology clinics in the North East of England. Potential subjects had been diagnosed with $\mathrm{MCl}$ in these services and were approached to take part in the Newcastle University LewyPro study [16]. Following consent, they had a detailed standardised neuropsychological assessment by a research nurse or psychologist,including the Addenbrooke's Cognitive Examination-Revised (ACE-R), FAS Verbal Fluency, the Trail-making Test Parts A and B, the Graded Naming Test (GNT) and the Rey Auditory Verbal Learning Test (AVLT). Full details are provided in Donaghy et al 2018 [17]. Subjects also underwent a thorough diagnostic medical assessment by a board-certified Old Age Psychiatrist (PCD), including blood sampling and neurological examination. Individuals were reviewed again one year later and the detailed clinical assessment was repeated. Diagnoses were confirmed as $\mathrm{MCl}-\mathrm{AD}$ by an expert consensus clinical panel (AJT, PCD, JPT) who reviewed all the clinical assessment data to confirm individuals met NIA-AA MCI criteria. Specifically, they had no clinical evidence of parkinsonism or of core symptoms of DLB [18]. This consensus panel diagnosis method has previously been validated against autopsy and is accepted by regulatory authorities as the clinical gold standard for living patients $[19,20]$. As Lewy body disease may be misdiagnosed as MCI-AD, we excluded any $\mathrm{MCl}-\mathrm{AD}$ individual with an abnormal FP-CIT scan from our control group. An abnormal FP$\mathrm{CIT}$ scan was defined by an abnormal consensus visual rating, given by a panel of five experts. Three of the $26 \mathrm{MCl}-A D$ individuals (aged 72, 76 and 79) were thus excluded because their FPCIT scans were rated as abnormal by the LewyPro study consensus panel of experts (PCD, JJL, GP, GR, AJT).

Thirty-one healthy controls were taken from a separate ongoing study recruiting healthy older people over 60 years old. They had undergone the same detailed neurological examination by a research physician as the individuals with $\mathrm{MCI}(\mathrm{RD}, \mathrm{SL})$, including rating for parkinsonism with UPDRS, a thorough neurocognitive examination which confirmed they had normal cognition and had a normal MRI brain scan. Again, they showed no evidence of clinical parkinsonism or of core symptoms of DLB. Incidental Lewy body disease is common in older people [21] so we excluded any healthy older adults with an abnormal rated FP-CIT scan from our control group to ensure that our data reflected age-related changes in adults without any 
evidence of Lewy body disease. Two of the 31 healthy older adults (aged 63 and 73) were thus excluded because their FP-CIT scans had been rated as abnormal by the expert panel (PCD, JJL, GP, JPT, AJT).

\section{Test group: individuals with dementia and $\mathrm{MCl}$}

Sixteen individuals over 60 years of age with probable DLB, 17 with probable AD, 20 with $\mathrm{MCl}$ due to probable Lewy body disease (MCl-LB) and 18 with $\mathrm{MCl}$ due to probable $A D$ (MCI-AD) were included to study the effect of age correction on FP-CIT results in older patients. Those with dementia were recruited as part of a Newcastle University study into the use of FP-CIT and cardiac MIBG in a representative UK population [22]. Those with $\mathrm{MCl}$ were recruited as part of an ongoing Newcastle University follow up study to the LewyPro study [16]. Note the MCI-AD individuals included here are not included in the above control group. As above, all individuals underwent a detailed neurological examination by a research physician, including rating for parkinsonism with UPDRS, and a thorough neurocognitive examination. Diagnoses were again made by a panel of three old-age psychiatrists (selected from AT, JK, PCD, JPT, JOB) blinded to scan results, based on clinical assessment and applying standard research criteria [1]. Baseline diagnoses were used for these groups as one year follow up diagnoses were not available.

\section{Image acquisition}

Individuals were scanned three to six hours following a bolus intravenous injection of 185 MBq of 123I-FP-CIT (DaTSCAN, GE Healthcare, UK) using a dual headed gamma camera (Siemens Symbia S or Siemens Intevo) fitted with low energy high resolution (LEHR) parallel hole collimators. One hundred and twenty 25 second views over a $360^{\circ}$ orbit (60 views per detector) were acquired on a $128 \times 128$ matrix with a zoom of $1.23 x$ giving a pixel size $3.9 \mathrm{~mm} \times 3.9 \mathrm{~mm}$. Overall image acquisition time was 25 minutes.

\section{Image processing and SBR calculation}

Image processing was performed on both a Hermes workstation (Hermes Medical Solutions, Stockholm, Sweden) with Hybrid Viewer BRASS v2.5 and using DaTQUANT v1.01 standalone software (GE Healthcare, Little Chalfont, UK). Both BRASS and DaTQUANT use a template derived from the FP-CIT and MRI scans of healthy controls to set striatal volumes of interest and occipital lobe (background) volumes. Both perform an automated image registration between the study loaded into the software and the template in order to automatically place the regions. Sub-regions such as the caudate, putamen and posterior putamen can be evaluated by both packages but we chose to focus on whole striatum results to reduce the effects of random noise due to small, possibly mis-registered volumes of interest in our data. Note that the Hybrid Viewer BRASS software is a major upgrade compared to "Classic BRASS" used in the ENC-DAT publication in 2013 [9]. There are also differences between the standalone DaTQUANT software and that supplied with GE's processing workstation Xeleris.

\section{BRASS:}

We obtained a calibration factor following a phantom method [23], which was applied to the SBR values within BRASS to correct for camera related systematic variations.

The raw patient data was reconstructed within Hermes HybridRecon Neurology, using OSEM iterative reconstruction (16 iterations and four subsets with a 3D Gaussian post filter of $0.7 \mathrm{~cm}$ ), 
with resolution recovery, uniform attenuation correction and Monte Carlo scatter correction. Reconstruction parameters specified by Hermes were used so that the BRASS normal database results, derived from images reconstructed with the same parameters, would be valid. The transaxial slice images were processed with BRASS to obtain left and right SBR values, age corrected Z scores and Z scores without age-correction.

\section{DaTQUANT:}

DaTQUANT does not have a camera correction feature because the PPMI multicentre study that the normal database scans were taken from did not take differences between gamma cameras into account. The raw FP-CIT data was imported into DaTQUANT and reconstructed within the program using OSEM iterative reconstruction with the default parameters used for the normal database ( 2 iterations and 10 subsets with a Butterworth filter of cut-off 0.6 cycles per $\mathrm{cm}$ and power 10), with no attenuation or scatter correction. The application of uniform attenuation correction within DaTQUANT was found to cause discontinuities between transverse slices in some cases with the default threshold applied. Recent studies have shown attenuation correction does not improve diagnostic accuracy [24, 25]; it was therefore not used with DaTQUANT.

SBR results and age corrected $Z$ scores for the left and right striatum were obtained for each individual. It is not possible to turn off age-correction when using the DaTQUANT normal database.

Analysis

A linear mixed effects model [26] (IBM SPSS v.24) was used to test for age dependency and any effect of gender, side (left vs right striatum) and dataset (MCI-AD vs adults with no cognitive impairment). Age, gender, side and dataset were input as fixed effects in the model and study ID input as a random effect. A mixed effects approach was used so that data from both striata could be included to give 104 SBR measurements; the random effect of study ID accounts for the fact that the SBR in the left and right striatum of an individual are likely to be correlated. The model was run for DaTQUANT and BRASS data separately, with both SBR and corrected Z score used as the dependent variable. P-values of less than 0.05 were taken to indicate effects significantly different from zero. The rate of change in SBR and corrected Z score with age and 95\% confidence limits were recorded.

\section{Results}

\section{Subject characteristics}

The age and sex of the individuals included and proportion of women in each group is given in Table 1.

\section{SBR age-dependency in the control group}

The mixed effects model showed that the effect of age, gender, striatum side or dataset ( $\mathrm{MCl}-\mathrm{AD}$ vs healthy adults) on SBR was not significant for either DaTQUANT or BRASS (see supplementary data). The only significant effect on age-corrected Z score was striatum side, 
which applied to DaTQUANT only, likely due to different slopes implemented in the software for right and left.

Plots of SBR against age for the 52 controls are shown in Figure 1 for the left striatum. Visually, there is no clear evidence of age-dependency in this age group. The slopes of the linear fits to the DaTQUANT and BRASS SBR data as a function of age for controls from the mixed effects model are given in Table 2 . The SBR slopes are negative but not significantly different from zero. The corrected $Z$ score slopes are larger and positive, however these are also not significantly different from zero.

$Z$ score results for our controls with age correction applied are shown plotted against age in Figure 2 (a) and (b) for BRASS and DaTQUANT. Both appear to show an increase in corrected $Z$ score with age, although the mixed effects model above showed that this was not statistically significant. Control $Z$ scores with the age correction function switched off in BRASS are plotted in Figure 3 (c). These $Z$ scores result from comparing to the mean SBR taken over the entire ENCDAT database age range. It is not possible to switch off age correction in DaTQUANT when using the factory installed PPMI database so we have not compared Z scores with and without age correction for DaTQUANT.

\section{Age correction in the test groups}

Figure 3 shows BRASS $Z$ scores with and without age correction for probable DLB, AD, MCI-LB and $\mathrm{MCl}-\mathrm{AD}$ (the test group). The lowest of the scores for the right and left striata have been taken, rather than one side or an average as this reflects clinical practice. The number of images correctly classified based on BRASS Z score values with and without age correction is shown in Table 3.

\section{Discussion}

Our results show that any decrease in the SBR of older adults with age appears to be very small, which suggests that a single linear fit over the entire adult age range may be inappropriate for older adults. The $95 \%$ confidence intervals for our linear fits (absolute SBR change per year) are -0.013 to +0.005 with DaTQUANT and -0.019 to +0.006 with BRASS. These correspond to percentage changes in SBR per decade in the control group of -7 to $+3 \%$ with DaTQUANT and -7 to $+2 \%$ with BRASS, taken over the range $70-80$ years. The confidence intervals encompass zero, and the linear mixed effects model showed no significant difference from zero in any measured slope, so our results do not support age-dependency in this age range. The current default SBR decreases modelled in the software correspond to approximately $-5 \%$ between 70 and 80 years for BRASS and -4 to $-7 \%$ for DaTQUANT, depending on striatal region. The slope between age and standardised uptake measurement (absolute change per year) reported in the 2017 metaanalysis was -0.032 [8], which was stated to correspond to a $-8.9 \%$ change per decade. This is an average over all decades so the percentage change per decade would be smaller for younger adults and greater for older adults, giving values much greater than our small estimates. We note that the 2007 study by Koch et al. concluding that the decline in dopamine transporter availability tails off in older age [12] was not included in the meta-analysis. This study reported 
an average decline of $9.5 \%$ per decade under 48 years of age and $6.3 \%$ on average over 48 years. This is evidence against a linear model, where the percentage effect would be expected to increase in magnitude with age. As was the case with the meta-analysis [8], Koch et al. [12] also reported similar $\mathrm{R}^{2}$ values for logarithmic and linear regression, suggesting that an exponential relationship is equally plausible. Yamamoto et al. [27] studied 30 healthy controls aged 50 to 86 years and reported a linear relationship between SBR and age (parameters not specified). However, they found that the percentage change in SBR per decade was lowest (2.4\%) for those over 70 years, which is not in keeping with a linear model.

We cannot exclude a small decrease in SBR with age, or indeed an increase, based on our dataset of 52 controls. However we note that the databases used by the manufacturers included similar numbers of participants over 60 years of age to our study (68 PPMI participants for DaTQUANT and around 55 ENC-DAT participants for BRASS). To our knowledge, no publications reporting a decrease in SBR with age have demonstrated that a statistically significant decrease also exists for older adults (>60 years), including the meta-analysis. A strength of our study lies in the focus on the age group most relevant to clinical practice.

Reviewing DaTQUANT database participants aged over 60 years within the software shows results similar to our controls, with no clear continuing decline in SBR in this age group. Visual review of published ENC-DAT control data also shows no definite decline in SBR with age beyond 60 years of age. However, the largest published dataset is the Japanese multicentre database [11], which does appear to show a continuing decline in SBR in older age. The SBR calculation method used in this study was the Southampton method [28], which unlike the commercial software does not attempt automated registration to a template. The Southampton method places large VOIs around each striatum to reduce partial volume effects and calculates SBRs based on an assumed striatal volume which is either kept the same for all patients or fixed values assigned depending on gender [29]. As the authors discussed, any loss of striatal volume with age will cause SBR to be underestimated with this method, possibly exaggerating the rate of decline with age [11]. Although it is possible to calculate SBR values for sub-striatal regions such as the caudate and putamen separately, we chose to consider only the whole striatum for this study. Since the caudate and putamen can usually not be fully distinguished on an FP-CIT scan, both BRASS and DaTQUANT have to use a somewhat arbitrary dividing line between them. In our experience, even when the putamen shows clearly reduced uptake visually it may have a normal SBR, due to counts from the caudate nucleus. In addition, with smaller regions, uncertainty due to lower counts is greater. The limitation of this is that the whole striatum analysis will be less sensitive to any losses occurring only in individual regions. It is known that putaminal uptake is often affected first in Lewy body disease and Nobili et al. [4] showed that for the ENC-DAT normal database the putamen to caudate ratio dropped with age. However their generalised linear model analysis showed no statistically significant difference between regions and so we conclude that any such effect is small and unlikely to explain our findings.

As with all such studies recruiting healthy controls, it is possible that the sample recruited is misrepresentative of the general population. This issue is likely to increase with age as a smaller proportion of older people may be able and willing to undergo the travel, tests and scans required to participate. We do not know whether older adults with above average general health are likely to have above average dopaminergic function but we cannot exclude this as a 
source of bias both for our study and the databases used in BRASS and DaTQUANT. Although we achieved a good gender balance within our control group, consisting of $44 \%$ women and $56 \%$ men, this was not the case for the $\mathrm{MCl}$ and dementia groups, predominantly due to the higher prevalence of Lewy body disease in males.

For the oldest adults (over 80 years of age) a drop in BRASS Z score of around one standard deviation is seen with age correction switched off. In our DLB dataset this would have changed the interpretation of the result from normal to abnormal in one case (from $Z=-1.42$ with age correction to -2.27 without). In our $\mathrm{MCl}$-LB dataset there are more borderline results and interpretation would have changed from normal to abnormal in three of the 20 cases. However, as our $A D$ and $\mathrm{MCl}-\mathrm{AD} Z \mathrm{Z}$ scores are slightly lower than expected with age correction applied, removing the age correction shifts $2 A D$ and three $M C I-A D$ cases below $Z$ scores of -2 . We note that turning age correction off in BRASS means that images are compared against the whole database, (mean age of 52), which exaggerates the effect somewhat as it is clear that an agedependency exists in younger adults. Ideally, we would compare our data against a subset of older database subjects only. Unfortunately it is not possible to recalculate a mean SBR using only the older ENC-DAT subjects as this data is not published or available within the software only the mean and standard deviation values for the whole dataset are used within BRASS. It is also not possible to adjust the DaTQUANT database to include only subjects over 60 as the original scans are required for creating a new database. At present, the only way of comparing SBRs against an older database without age correction is to set up a local control database.

In summary, we found that the rate of change in SBR with age for control subjects over 60 was not significantly different from zero, suggesting that any decline in SBR with age in healthy older adults is likely to be lower than previously thought. Both BRASS and DaTQUANT apply slopes that are greater than our estimates, leading to potentially large differences between the software age-adjusted SBR and individual values for the oldest patients. With BRASS, Z scores are around 1SD lower with age correction switched off for subjects over 80 years of age. Use of current age correction methods in older people being assessed for DLB and MCI-LB could contribute to false negative reporting and misdiagnosis and therefore caution is suggested in clinical practice and research applications.

\section{Acknowledgements}

The authors would like to thank Miss Helen Kain, Research Support Secretary and our colleagues in the Nuclear Medicine department at the Newcastle Royal Victoria Infirmary. We thank the Alzheimer's Society for supporting the Fellowship of the lead author and the NIHR and Alzheimer's Research UK for supporting the clinical research studies used in this project. We are grateful to Liz Clarke (GE Healthcare) and Helena McMeekin (Hermes Medical) for providing information on DaTQUANT and BRASS respectively.

\section{Financial Disclosure / Conflict of Interest}

${ }^{123}$ I-FP-CIT radiopharmaceutical and the DaTQUANT software package were provided by GE Healthcare. We have no other conflicts of interest relevant to this study. 


\section{References}

1. McKeith, I.G., et al., Diagnosis and management of dementia with Lewy bodies: Fourth consensus report of the DLB Consortium. Neurology, 2017. 89(1): p. 88-100.

2. Koch W, et al., Clinical testing of an optimized software solution for an automated, observer independent evaluation of dopamine transporter SPECT studies. J Nucl Med, 2005. 46(7): p. 10.

3. Calvini, P., et al., The basal ganglia matching tools package for striatal uptake semiquantification: description and validation. Eur J Nucl Med Mol Imaging, 2007. 34(8): p. 1240-53.

4. Nobili, F., et al., Automatic semi-quantification of [123I]FP-CIT SPECT scans in healthy volunteers using BasGan version 2: results from the ENC-DAT database. Eur J Nucl Med Mol Imaging, 2013. 40(4): p. 565-73.

5. Pencharz, D.R., et al., Automated quantification with BRASS reduces equivocal reporting of DaTSCAN (123I-FP-CIT) SPECT studies. Nucl Med Rev Cent East Eur, 2014. 17(2): p. 65-9.

6. Varrone, A., et al., European multicentre database of healthy controls for [123I]FP-CIT SPECT (ENC-DAT): age-related effects, gender differences and evaluation of different methods of analysis. Eur J Nucl Med Mol Imaging, 2013. 40(2): p. 213-27.

7. Booij, J., et al., Diagnostic Performance of the Visual Reading of (123)I-Ioflupane SPECT Images With or Without Quantification in Patients With Movement Disorders or Dementia. J Nucl Med, 2017. 58(11): p. 1821-1826.

8. Karrer, T.M., et al., Reduced dopamine receptors and transporters but not synthesis capacity in normal aging adults: a meta-analysis. Neurobiol Aging, 2017. 57: p. 36-46.

9. Varrone A, et al., European multicentre database of healthy controls for [123I]FP-CIT SPECT (ENC-DAT): age-related effects, gender differences and evaluation of different methods of analysis. . Eur J Nucl Med Mol Imaging, 2013. 40(2): p. 15.

10. van Dyck, C.H., et al., Age-related decline in dopamine transporters: analysis of striatal subregions, nonlinear effects, and hemispheric asymmetries. Am J Geriatr Psychiatry, 2002. 10(1): p. 36-43.

11. Matsuda, H., et al., Japanese multicenter database of healthy controls for [(123)I]FPCIT SPECT. Eur J Nucl Med Mol Imaging, 2018. 45(8): p. 1405-1416.

12. Koch, W., et al., Extended studies of the striatal uptake of 99mTc-NC100697 in healthy volunteers. J Nucl Med, 2007. 48(1): p. 27-34.

13. Koch, W., et al., Extrastriatal binding of [(1)(2)(3)I]FP-CIT in the thalamus and pons: gender and age dependencies assessed in a European multicentre database of healthy controls. Eur J Nucl Med Mol Imaging, 2014. 41(10): p. 1938-46.

14. The Parkinson Progression Marker Initiative (PPMI). Prog Neurobiol, 2011. 95(4): p. 629-35.

15. Albert, M.S., et al., The diagnosis of mild cognitive impairment due to Alzheimer's disease: recommendations from the National Institute on Aging-Alzheimer's Association workgroups on diagnostic guidelines for Alzheimer's disease. Alzheimers Dement, 2011. 7(3): p. 270-9. 
16. Thomas, A.J., et al., Diagnostic accuracy of dopaminergic imaging in prodromal dementia with Lewy bodies. Psychol Med, 2018: p. 1-7.

17. Donaghy, P.C., et al., Neuropsychiatric symptoms and cognitive profile in mild cognitive impairment with Lewy bodies. Psychol Med, 2018. 48(14): p. 2384-2390.

18. Thomas, A.J., et al., Autopsy validation of 123I-FP-CIT dopaminergic neuroimaging for the diagnosis of DLB. Neurology, 2017. 88(3): p. 276-283.

19. McKeith I, et al., Sensitivity and specificity of dopamine transporter imaging with (123)I-FP-CIT SPECT in dementia with Lewy bodies: a phase III, multicentre study. 2007.

20. McKeith, I., et al., Predictive Accuracy of Clinical Diagnostic Criteria for Dementia with Lewy Bodies: A Prospective Neuropathological Validation study. 54, 1050-1058. Neurology, 2000(54): p. 1050-1058.

21. Markesbery, W.R., et al., Lewy body pathology in normal elderly subjects. J Neuropathol Exp Neurol, 2009. 68(7): p. 816-22.

22. Kane, J.P.M., et al., (123)I-MIBG scintigraphy utility and cut-off value in a clinically representative dementia cohort. Parkinsonism Relat Disord, 2019.

23. Tossici-Bolt, L., et al., Calibration of gamma camera systems for a multicentre European I-123-FP-CIT SPECT normal database. European Journal of Nuclear Medicine and Molecular Imaging, 2011. 38(8): p. 1529-1540.

24. Akahoshi, M., et al., Attenuation and scatter correction in I-123 FP-CIT SPECT do not affect the clinical diagnosis of dopaminergic system neurodegeneration. Medicine (Baltimore), 2017. 96(45): p. e8484.

25. Dickson JC, T.-B.L., Sera T, Booij J, Ziebell M, Morbelli S,, B.T. Assenbaum-Nan S, Pagani M, Kapucu OL, Hesse S, Van Laere K, Darcourt, and V.A. J, Tatsch K, The impact of reconstruction and scanner characterisation on the diagnostic capability of a normal database for [(123)I]FP-CIT SPECT imaging. Ejnmmi Research, 2017. 7(1): p. 10.

26. Pinheiro, J.C. and D.M. Bates, Mixed-Effects Models in S and S-PLUS. 2000: Springer Science \& Business Media. 528.

27. Yamamoto, H., et al., Age-related effects and gender differences in Japanese healthy controls for [(123)I] FP-CIT SPECT. Ann Nucl Med, 2017. 31(5): p. 407-412.

28. Tossici-Bolt, L., et al., Quantification of [123I]FP-CIT SPECT brain images: an accurate technique for measurement of the specific binding ratio. Eur J Nucl Med Mol Imaging, 2006. 33(12): p. 1491-9.

29. Tossici-Bolt L, et al., Quantification of [123I]FP-CIT SPECT brain images: an accurate technique for measurement of the specific binding ratio. . Eur J Nucl Med Mol Imaging, 2006. 33(12). 
Figures
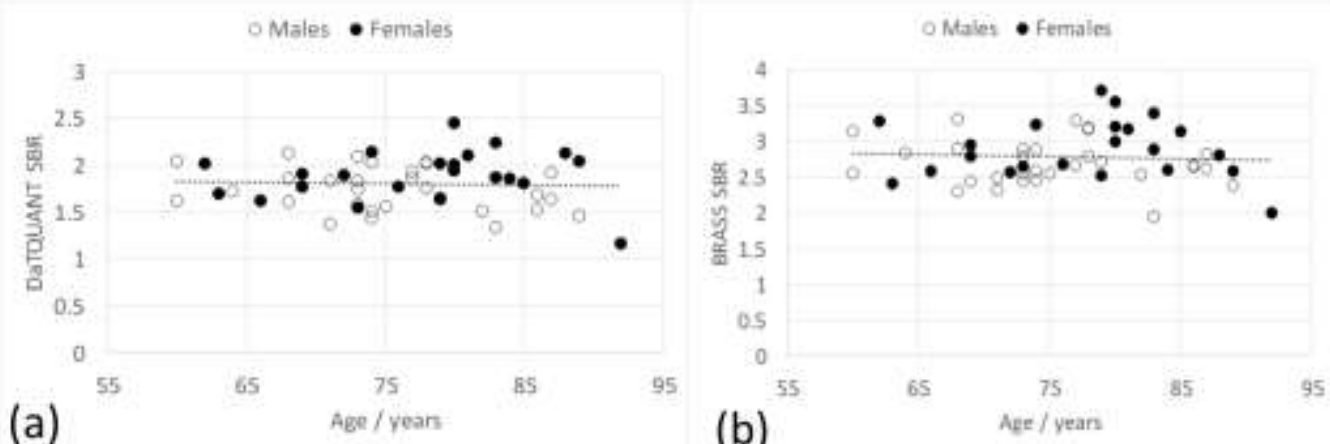

(a)

Age / year

(b)

Lee / year

Figure 1: Mean striatum SBR values plotted against age for DaTQUANT (a) and BRASS (b). Values for females are shown in solid circles, males in open circles.
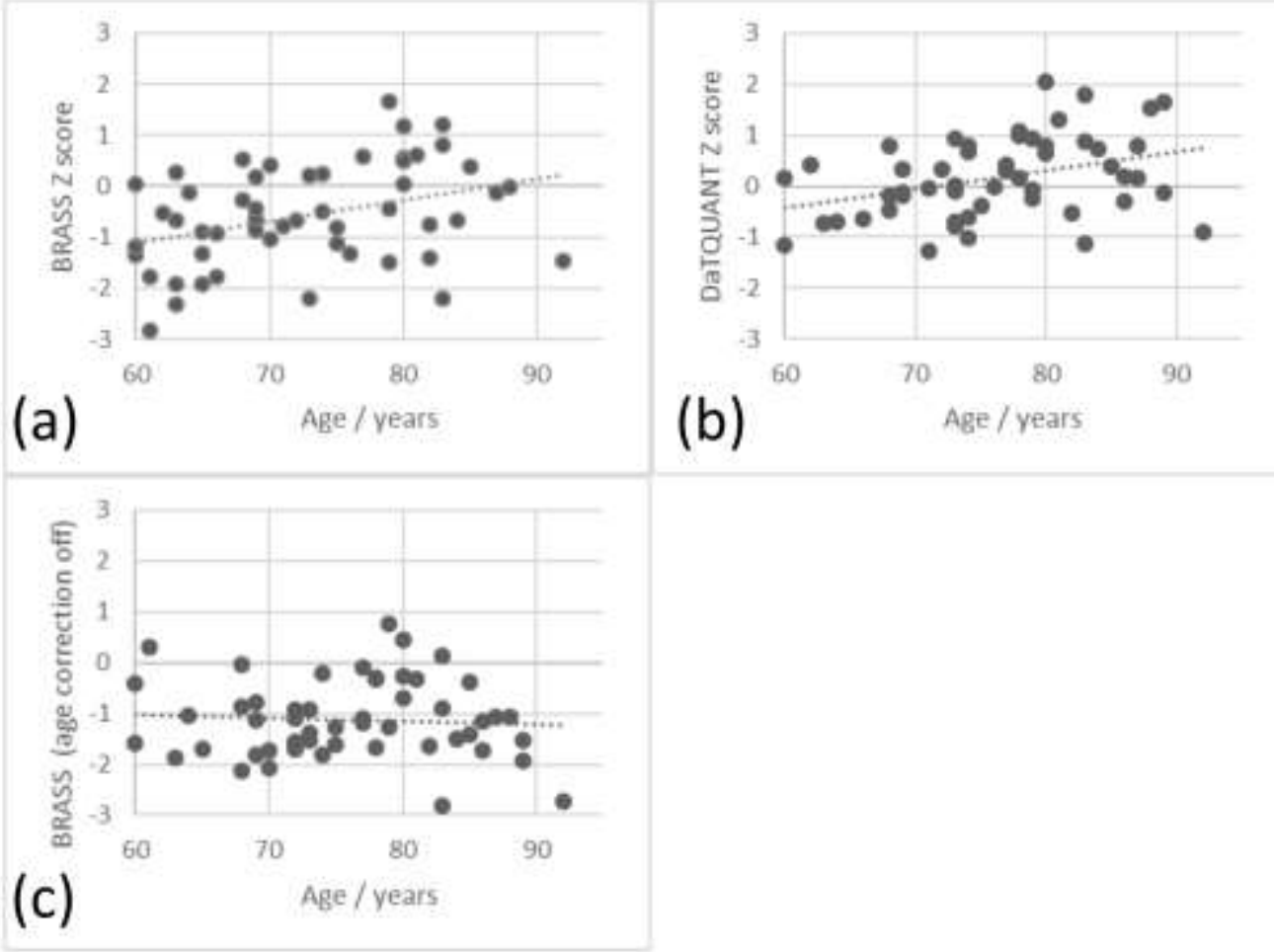

Figure 2: BRASS Z scores (lowest of left and right striatum) with and without age correction for probable DLB (a) probable AD (b), probable MCI-LB (c) and probable MCI-AD (d). 


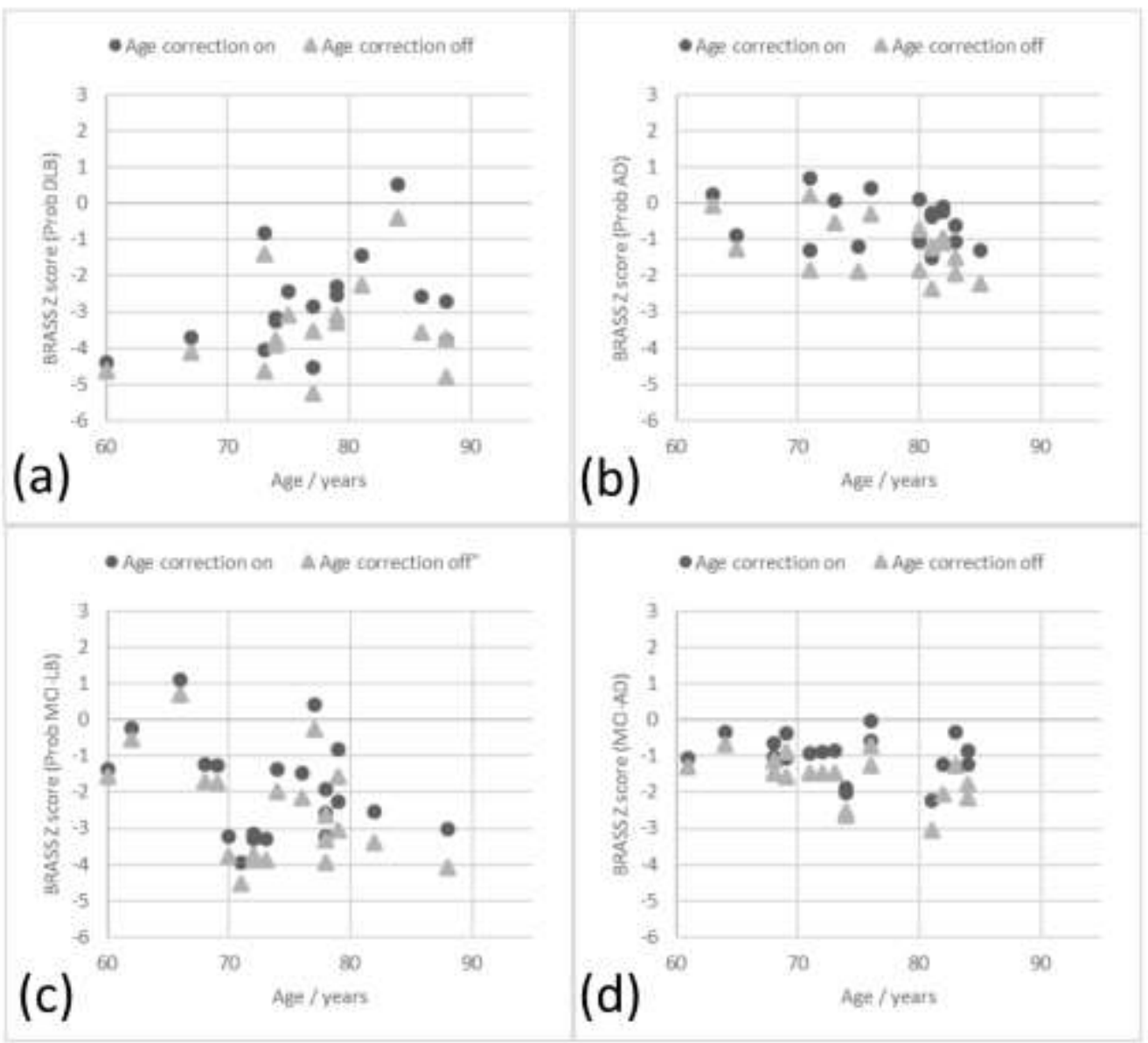

Figure 3: (a) BRASS age corrected Z scores for controls, (b) DaTQUANT age corrected $Z$ scores and (c) BRASS $Z$ scores without age correction. The average values of the right and left striatum SBRs are used. 


\section{Tables}

Table 1: Ages and gender balance of the individuals with $M C l$ due to $A D$, the healthy older adults, the two groups taken together as a control group and the test individuals with DLB, $A D$, $\mathrm{MCl}-\mathrm{LB}$ and $\mathrm{MCl}-\mathrm{AD}$.

\begin{tabular}{|l|l|c|c|l|}
\hline \multirow{4}{*}{ Controls } & & $\begin{array}{l}\text { Number } \\
\text { included }\end{array}$ & Mean age (SD) & $\begin{array}{l}\text { Proportion } \\
\text { female }\end{array}$ \\
\cline { 2 - 5 } & Probable MCl-AD & 23 & $77.7(8.3)$ & $0.61(14 / 23)$ \\
\cline { 2 - 5 } & $\begin{array}{l}\text { Healthy older } \\
\text { adults }\end{array}$ & 29 & $74.5(7.6)$ & $0.28(8 / 29)$ \\
\cline { 2 - 5 } & Total controls & 52 & $75.9(8.0)$ & $0.42(22 / 52)$ \\
\hline \multirow{5}{*}{ Test group } & Probable DLB & 16 & $77.2(7.5)$ & $0.06(1 / 16)$ \\
\cline { 2 - 5 } & Probable AD & 17 & $77.2(6.5)$ & $0.24(4 / 17)$ \\
\cline { 2 - 5 } & Probable MCI-LB & 20 & $73.6(6.8)$ & $0.10(2 / 20)$ \\
\cline { 2 - 5 } & $\begin{array}{l}\text { Probable MCI-AD } \\
\text { (test group) }\end{array}$ & 18 & $73.8(6.9)$ & $0.44(8 / 18)$ \\
\cline { 2 - 5 } & Total test group & 71 & $\mathrm{n} / \mathrm{a}$ & $\mathrm{n} / \mathrm{a}$ \\
\hline
\end{tabular}

Table 2: Rate of change in SBR and age corrected Z score per year for Newcastle control subjects over 60 years of age calculated with DaTQUANT and BRASS

SBR

\begin{tabular}{|c|c|c|c|c|c|c|}
\hline & Slope & $\begin{array}{c}\text { 95\% confidence } \\
\text { interval }\end{array}$ & $p$-value & Slope & $\begin{array}{c}\text { 95\% confidence } \\
\text { interval }\end{array}$ & $\begin{array}{c}\mathrm{p}- \\
\text { value }\end{array}$ \\
\hline DaTQUANT & -0.004 & -0.013 to 0.005 & 0.35 & 0.017 & -0.009 to 0.043 & 0.19 \\
\hline BRASS & -0.007 & -0.019 to 0.006 & 0.30 & 0.015 & -0.011 to 0.041 & 0.26 \\
\hline
\end{tabular}

\section{Age-corrected Z score}

Table 3: Number of individuals with BRASS age corrected and non-corrected Z scores above and below our threshold of -2SD.

\begin{tabular}{|l|c|c|c|c|c|c|}
\hline & \multicolumn{3}{|c|}{ BRASS age correction on } & \multicolumn{3}{c|}{ BRASS age correction off } \\
\hline & $\begin{array}{l}\text { Z below } \\
-2\end{array}$ & $\begin{array}{l}\text { Z above } \\
-2\end{array}$ & $\begin{array}{l}\text { \% with expected } \\
\text { Z score }\end{array}$ & $\begin{array}{l}\text { Z below - } \\
2\end{array}$ & $\begin{array}{l}\text { Z above } \\
-2\end{array}$ & $\begin{array}{l}\text { \% with expected } \\
\text { Z score }\end{array}$ \\
\hline Controls & 4 & 48 & $92 \%$ & 4 & 48 & $92 \%$ \\
\hline Prob DLB & 13 & 3 & $81 \%$ & 14 & 2 & $88 \%$ \\
\hline Prob AD & 0 & 17 & $100 \%$ & 2 & 15 & $88 \%$ \\
\hline Prob MCI-LB & 10 & 10 & $50 \%$ & 13 & 7 & $65 \%$ \\
\hline Prob MCl-AD & 2 & 16 & $89 \%$ & 5 & 13 & $72 \%$ \\
\hline
\end{tabular}


Supplementary data:

Estimate of fixed effects from SPSS mixed effects model for DaTQUANT and BRASS for dependent variables SBR and Z score.

\begin{tabular}{|c|c|c|c|c|c|}
\hline & & Striatal bindi & atio (SBR) & SBR Z score & \\
\hline Co-variate & & DaTQUANT & BRASS & DaTQUANT & BRASS \\
\hline Age & Estimate & -0.041 & -0.066 & 0.170 & 0.015 \\
\hline & T statistic & -0.947 & -1.048 & 1.328 & 1.142 \\
\hline & $\begin{array}{l}95 \% \\
\text { confidence } \\
\text { interval }\end{array}$ & $\begin{array}{l}-0.013 \text { to } \\
+0.005\end{array}$ & $\begin{array}{l}-0.019 \text { to } \\
+0.006\end{array}$ & $\begin{array}{l}-0.009 \text { to } \\
0.427\end{array}$ & $\begin{array}{l}-0.011 \text { to } \\
+0.041\end{array}$ \\
\hline & $p$-value & 0.348 & 0.300 & 0.190 & 0.259 \\
\hline Gender & Estimate & 0.136 & 0.149 & 0.396 & 0.309 \\
\hline & T statistic & 1.868 & 1.419 & 1.865 & 1.443 \\
\hline & $\begin{array}{l}95 \% \\
\text { confidence } \\
\text { interval }\end{array}$ & $\begin{array}{l}-0.010 \text { to } \\
+0.283\end{array}$ & $\begin{array}{l}-0.062 \text { to } \\
+0.361\end{array}$ & $\begin{array}{l}-0.310 \text { to } \\
+0.824\end{array}$ & $\begin{array}{l}-0.121 \text { to } \\
+0.739\end{array}$ \\
\hline & $p$-value & 0.068 & 0.162 & 0.068 & 0.155 \\
\hline Control & Estimate & 0.090 & 0.205 & 0.251 & 0.430 \\
\hline & T statistic & 1.238 & 1.951 & 1.185 & 2.018 \\
\hline & $\begin{array}{l}95 \% \\
\text { confidence } \\
\text { interval }\end{array}$ & $\begin{array}{l}-0.056 \text { to } \\
+0.237\end{array}$ & $\begin{array}{l}-0.006 \text { to } \\
+0.416\end{array}$ & $\begin{array}{l}-0.175 \text { to } \\
+0.678\end{array}$ & $\begin{array}{l}+0.001 \text { to } \\
+0.859\end{array}$ \\
\hline & $p$-value & 0.222 & 0.057 & 0.242 & 0.049 \\
\hline Striatum & Estimate & 0.006 & 0.022 & 0.159 & 0.002 \\
\hline & T statistic & 0.429 & 1.152 & 3.372 & 0.049 \\
\hline & $\begin{array}{l}95 \% \\
\text { confidence } \\
\text { interval }\end{array}$ & $\begin{array}{l}-0.022 \text { to } \\
+0.034\end{array}$ & $\begin{array}{l}-0.016 \text { to } \\
+0.060\end{array}$ & $\begin{array}{l}+0.064 \text { to } \\
+0.254\end{array}$ & $\begin{array}{l}-0.077 \text { to } \\
+0.0810\end{array}$ \\
\hline & $p$-value & 0.669 & 0.255 & 0.001 & 0.961 \\
\hline
\end{tabular}

